\title{
Radionoticieros, información política y proceso electoral. Un acercamiento desde la perspectiva de los usuarios'
}

Francisco de Jesús Aceves González Departamento de Estudios de la Comunicación Social, Universidad de Guadalajara

\begin{abstract}
Cov
ON LA IRRUPCIÓN en el escenario político nacional de contiendas electorales más competitivas, el papel de los medios masivos de comunicación como transmisores de información política ha experimentado un sensible redimensionamiento que ha impactado en forma sustancial, a los diversos actores que inciden en el proceso de la comunicación política: partidos, medios informativos y opinión pública. Los estrategas de las campañas políticas reconocen a los medios masivos como el principal instrumento de difusión de las propuestas y la imagen de sus candidatos. Los mismos medios son impactados por el evento electoral en la conformación de su oferta programática, en particular la relacionada con el cumplimiento de su función informativa, com en el caso de los radionoticieros. Finalmente, la opinón pública los utiliza como la principal fuente de información sobre los asuntos políticos.

Las funciones informativas de la radio, como vehículo de difusión de acontecimientos políticos, se remontan a los albores de su irrupción como medio de comunicación masiva, cuando en el año de 1920 la KDKA transmitió en directo el resultado de las elecciones presidenciales estadounidenses escenificadas por Harding y Cox. Desde su inicio se advertía que por sus características tecnológicas la radio como medio informativo superaba ampliamente a la prensa escrita en el terreno de la inmediatez, aunque no en la amplitud de la oferta noticiosa y en la profundidad de su tratamiento. No obstante el surgimiento de programas informativos producidos específicamente para la radiodifusión aparecería algunos lustros más tarde.
\end{abstract}

1 El autor agradece profundamente a las personas que participaron en la aplicación de las encuestas y de manera especial a Guadalupe García Pérez por su colaboración en la implementación de los sondeos y en la captura de información. 
Radionoticieros, información política y proceso electoral ...

En el caso de Jalisco, el inicio de los servicios informativos proporcionados por la radio se encontraban vinculados estrechamente con la prensa escrita. En efecto, los periódicos suministraban la materia prima con que se confeccionaban los programas noticiosos. El resultado era la transmisión resumida de las notas que a consideración del productor-locutor tuvieran cierta relevancia. Los esfuerzos dirigidos a la creación de programas informativos producidos por las empresas radiofónicas constituían excepciones. Una de las mas notables fué la realizada por el periodista Ildefonso Loza Márquez, impulsor del programa "Gaceta del Aire" que se difundía por una estación local. ${ }^{2}$

Sin embargo, el verdadero auge de los radionoticieros ocurriría hasta la década de los setenta, cuando aparecen en la barra matutina de la radio diversos programas de noticias, además de que estaciones completas adoptan un perfil informativo. En julio de 1974 INFORJAL (Notisistema en la actualidad) inicia sus transmisiones ${ }^{3}$, posteriormente Radio Metrópoli adopta una programación conformada por diversos productos de corte informativo.

A principios de los setenta, en un sondeo realizado por la UNAM, un porcentaje significativo de los radioescuchas tapatíos señalaba a los noticieros como uno de los dos tipos de programas que prefería de la radio. En efecto, el rubro "noticieros" resultó el elegido después del de "musicales" por el $22 \%$ de los radioescuchas del estrato "A/B"; el $20 \%$ del estrato " $\mathrm{C}$ " y el $16 \%$ del "D". ${ }^{4}$ En la actualidad, la información radiofónica rivaliza entre las preferencias del auditorio con la prensa escrita, aunque ambas son superadas ampliamente por la televisión.

En el presente artículo se analiza, precisamente, la oferta informativa de los radionoticieros desde la perspectiva de sus radioescuchas en el marco del proceso electoral de 1994 en la ciudad de Guadalajara, específicamente de diciembre de 1993 a agosto de 1994. Se trata pues, de definir algunas de las características más relevantes del público

2 Cinthya Lizette Garcia de Quevedo, La gaceta del aire, Tesis de licenciatura, UNIVA, 1992.

3 María Elena Hernández, "La producción de noticias en una organización radiofónica tapatía: Notisistema" en Radiodifusión Regional enMéxico. Historias. Programas. Audiencias. Fco. de J.Aceves etaal, (comps), Universidad de Guadalajara, México, 1991.

4 Encuesta nacional sobre radio y televisión. Facultad de Comercio y Administración, UNAM, 1971. Este estudio constituye el primer esfuerzo académico por documentar algunas de las características de la audiencia de los medios masivos en México. A pesar de sus deficiencias, ofrece valiosos datos que permiten definir un cierto perfil de los usuarios de los medios, tanto a nivel nacional como en cada una de las 15 ciudades que integraron la muestra. Concretamente en Guadalajara, los datos que permiten la caracterización de la audiencia se sustentan en los resultados obtenidos tras la aplicación de 3,377 encuestas. 
usuario de la oferta informativa de los radionoticieros. ¿Cuáles son sus patrones de exposición?, ¿Cuál es su valoración de los radionoticieros como medios de información política?, ¿Cómo evalúan la información que éstos suministraron con respecto al desarrollo de la contienda electoral?

El trabajo se sustenta en la información empírica basada en los resultados obtenidos de tres encuestas, aplicadas a lo largo del proceso electoral. Las fechas en que se levantaron (diciembre 1993, abril y agosto de 1994) corresponden a momentos claves del proceso: la difusión pública de los contendientes a la presidencia; el punto intermedio entre el arranque de las campañas y la fecha de los comicios; y los días previos a la votación. $^{5}$

\section{La oferta informativa de la radiodifusión}

En un estudio realizado a finales de los ochenta ${ }^{6}$, (Aceves, 1988) Jalisco ocupaba el segundo lugar, a nivel nacional, respecto al número de estaciones radiofónicas asentadas en su territorio. En efecto, 62 radioemisoras se diseminaban en 15 localidades a lo largo y ancho del estado. Sin embargo, 43 de ellas (69.4\%) se concentraban en la zona metropolitana de Guadalajara. De éstas 24 transmitian en la banda de amplitud modulada (AM) y 19 en la de frecuencia modulada (FM). Para diciembre de 1993 el número de emisoras en la zona metropolitana se había incrementado a 47, -26 en la banda de AM y 21 en la de FM-, y en agosto de 1994 se había adicionado una estación más en la banda de $\mathrm{FM}^{7}$

A diferencia de los demás medios de comunicación, la oferta radiofónica disponible para los radioescuchas tapatíos se conformaba casi exclusivamente por los programas transmitidos por estaciones emisoras de la localidad. Si bien es cierto, que en el espacio jalisciense transitan un número incalculable de ondas electromagnéticas que son portadoras de señales emitidas por incontables estaciones, las condiciones tecnológicas para la decodificación-recepción de tales señales, restringen el acceso a solamente aquellas cuyas condiciones de transmisión (lugar y potencia) hacen posible su adecuada sintoni-

5 Para mayor información sobre las muestras ver Radiografía de la Muestra en el Anexo.

6 Francisco de Jesús Aceves, "Radiodifusoras en Jalisco. Cuántas son..." en Revista Mexicana de Comunicación, año I, núm. 1, septiembre-octubre 1988.

7 Medios Audiovisuales, Medios Publicitarios Mexicanos S.A. de C.V., diciembre 1993 y septiembre 1994. 
Radionoticieros, información política y proceso electoral ...

zación. Lo que significa que son las emisoras avencidadas en la localidad las que umplen adecuadamente con dichas condiciones.

Empero, esta determinación técnica no significa que el contenido programático de las emisoras se concrete al productos generados en el ámbito local, sino que algunos programas -especialmente de carácter informativo- que se difunden por estaciones locales son confeccionados en otro lugar, básicamente en la capital del país. Esta situación se explica en el contexto de la evolución histórica de la industria radiofónica nacional, donde el surgimiento de grandes consorcios y la conformación de cadenas a nivel nacional son las características distintivas del modelo radiofónico mexicano. En rigor, la totalidad de las emisoras comerciales pertenecen a alguno de estos agrupamientos -cadenas-de carácter nacional. Esta situación tiene consecuencias en el campo de la programación, especialmente en el relacionado con los productos informativos y más concretamente con los radionoticieros.

En efecto, durante el período analizado, los radioescuchas tapatios podían optar por una amplia gama de servicios informativos, que de acuerdo a sus características particulares, podrían clasificarse en dos tipos: los radionoticieros y las estaciones informativas.

En cl caso de los radionoticieros, se trata de productos informativos cuyo contenido básico es la presentación de noticias de actualidad. Generalmente cuentan con una emisión estelar que se difunde en las primeras horas de la mañana cuya duración fluctúa entre las 3 y las 4 horas. Algunos de éstos programas cuentan con emisiones al mediodía y en la tarde y otros ofrecen resúmenes informativos a lo largo del dia. Una característica común en este tipo de programas es la presencia de conductores titulares en las emisiones estelares, así como en que el formato de los mismos presenta más similitudes que diferencias. Un caso especial lo constituye Notisistema el cual no cuenta con programa estelar y sus servicios informativos adoptan un formato más parecido al resumen informativo. En este rubro de radionoticieros se encontraban, el mencionado Notisistema, Notisat, Aqui Guadalajara -que en el transcurso del proceso cambió su denominación a más noticias-, Notiexpress y Línea Universitaria, confeccionados en la localidad, además de Monitor, Informativo Panorama, Para empezar y Buenos Días que se producian en la ciudad de México.

En el caso de las estaciones informativas se trata de estaciones cuya programación se integraba casi en su totalidad por productos de contenido informativo de muy diverso género. En su barra programática alternaban programas de información especializada (deportiva, financiera, legal, doméstica, etc.) con radionoticieros. Era el caso de Rario Metrópoli (XEAD) estación base de Notisistema que transmitía noticias, entrevistas e 
información en general las 24 horas del día, Radio Red Guadalajara (XEDKR) que transmitía simultáneamente con XERED de la ciudad de México -estación sede de Monitor-, La Triple AAA (XEAAA) que transmitía también las 24 horas programas noticiosos e informativos de interés local y regional, XEDK -estación base de Aquí Guadalajara- que alternaba los programas informativos con musicales y XEWK, con amplia programación de corte informativo y que difundía para los radioescuchas el telenoticiero 24 Horas, que a nivel nacional transmitía el canal 2 de Televisa.

Otro elemento a considerar es la presencia de los programas informativos en las estaciones de radio, así como su penetración entre la radioaudiencia. Un estudio realizado en 1981, detectaba que de las 810 estaciones radiofónicas que se encontraban en funcionamiento a lo largo del país, solamente 113 (13.9\%) transmitián noticieros como parte de su programación (BOHMANN, 1989). ${ }^{8}$ Esta raquítica participación de programas informativos acusó una notable modificación como resultado del "boom" de los radionoticieros experimentado a fines de los setenta y principios de los ochenta. En el caso de Guadalajara, hacia 1990, de las 47 emisoras que funcionaban en el área metropolitana 25 (53.2\%) contaban en su programación con productos informativos. De éstas, 15 transmitían en la banda de AM y las restantes en la de FM. Sin embargo la aparente amplitud de estaciones con servicios informativos se reduce sustancialmente si se considera que solamente tres radionoticieros representaban la oferta informativa de 16 de las 25 emisoras ( 9 en AM y 7 en FM). En efecto, Notisistema con difusión regional se transmitía por XEAD, XEAD-FM, XEQP, XETIA, XETIA-FM y XHOY; Notisat producto informativo de Promosat. S.A., con difusión a nivel nacional e internacional, formaba parte de la programación de las estaciones XEAAA, XEBBB, XECCC, XHBIO, XHGEO X XHRA; finalmente Más Noticias, radionoticiero especificamente local, se difundía por XEDK, XEDKT, XEDKR (enlazado a Radiored y que retransmitía Monitor y XHDK.

Acerca de su penetración en la radioaudiencia, a la fecha no existe en la literatura disponible datos confiables en este sentido. Aunque en el sondeo aplicado por Aceves (1991) se detectó que de las dieciocho radiodifusoras que captaban el $80 \%$ de las preferencias, 10 se encontraban en la banda de AM y 8 en la de $\mathrm{FM}^{9}{ }^{9}$ lo que indica una

8 Karin Bohmann. Medios de comunicación y sistemas informativos en México, CNCAAlianza Editorial Mexicana, México, 1989.

9 Francisco de Jesús Aceves. "Las estaciones preferidas de los radioescuchas tapatíos" en Radiodifusion Regional en México. Historias, programas, audiencias, Aceves, Arredondo y Luna (comps), Universidad de Guadalajara, México, 1991. 
distribución casi similar hacia las dos opciones, no se registra la exposición de los usuarios hacia los servicios informativos de la radio. Uno de los propósitos del presente estudio es precisamente subsanar en parte, este vacío en el conocimiento.

\section{Patrón de exposición y hábitos de consumo del periodismo radiofónico}

Un primer dato que resulta sorpresivo al analizar los patrones de exposición hacia los radionoticieros, es que el porcentaje de entrevistados que aceptó escuchar eventualmente algún radionoticiero resultó inferior a los que aceptaron exponerse a los otros medios, tanto en el sondeo de diciembre de 1993 (58.5\%) como en el de agosto de 1994 (57.2\%). Si se considera que la radio es el medio de mayor penetración rivalizando en este campo con la misma televisión, como lo demuestra el hecho de que en 1991, de una muestra de 703 entrevistados 664 (94.5\%) se reconocieron como radioescuchas (Aceves, ibid: 194), resulta sorprendente la escasa proporción de radioescuchas que se exponen a programas de contenido informativo. Esto significa que en tanto vehículo informativo la radiodifusión se encuentra a la zaga de la prensa escrita y la televisión.

Otros datos que los reultados de los sondeos ponen en evidencia, es que el patrón de exposición presenta características específicas al ser analizado desde las diversas variables de estrato socioeconómico, género, edad, escolaridad y actividad ocupacional.

Con respecto al estrato los sondeos presentan variaciones importantes en los patrones de exposición de los radioescuchas. Las diferencias se expresan tanto en los distintos sondeos como en las proporciones de los radioescuchas pertenecientes a cada estrato. En el sondeo de diciembre, los radiooyentes del estrato " $\mathrm{D}$ " resultaron ser quienes proporcionalmente obtuvieron en mayor índice de exposición, pero en el sondeo de agosto registraron el menor. Por otra parte, los entrevistados del estrato " $\mathrm{A}$ " que en diciembre rebasaban apenas el $50 \%$, en agosto manifestaron un impresionante crecimiento de casi treinta puntos porcentuales. En los estratos restantes, se aprecia también un significativo incremento. En este caso, se podría inferir, a partir de estos datos, que la cercanía del evento electoral tuvo que ver en el incremento de usuarios de radionoticieros, y de que dicho incremento se hizo más evidente solamente en los estratos " $\mathrm{A}$ " y " $\mathrm{B}$ " que son los de mayor ingreso. 


\section{Cuadro 1}

Usuarios de radionoticieros por estrato social

(porcentajes)

\begin{tabular}{|l|c|c|c|c|}
\hline & A & B & C & D \\
\hline Diciembre 1993 & 52,2 & 46.9 & 57,9 & 62,0 \\
\hline Agosto 1994 & 80,9 & 57,1 & 64,0 & 51,7 \\
\hline
\end{tabular}

Fuente: Sondeos diciembre 1993 y agosto 1994

Con relación a la variable "género", el patrón de exposición entre los usuarios de los radionoticieros no registra diferencias notables entre ambos sexos. Lo que se observa es una proporción casi similar entre los entrevistados de cada sexo que aceptaron escuchar algún radionoticiero. En rigor, se aprecia un patrón casi homogéneo en ambos sondeos. En este caso, la cercanía de las elecciones solamente registra una variación de tipo negativo en el patrón de exposición observado por el sexo femenino, que no resulta significativo, en contraste con el pequeñísimo incremento experimentado entre los varones, lo que podría ser interpretado como un mayor interés de éstos hacia el evento electoral.

\section{Cuadro 2}

Usuarios de radionoticieros por género

(porcentajes)

\begin{tabular}{|l|c|c|}
\hline & Femenino & Masculino \\
\hline Diciembre 1993 & 59,6 & 57,2 \\
\hline Agosto 1994 & 56,7 & 57,9 \\
\hline
\end{tabular}

Fuente: Sondeos diciembre 1993 y agosto 1994

En el caso de la edad, se observa también cierta homogeneidad en los patrones de exposición de los diferentes grupos etarios. Los mayores índices los alcanzan quienes se ubican entre los 26 y 35 años de edad, seguido por los de 36 a 50. En ambos casos, la proporción se incrementa en el sondeo de agosto, en contraste con los grupos restantes que experimentan una disminución. En este caso las proximidad de las elecciones parece haber estimulado la exposición del sector de radioescuchas que se encuentran entre los 26 y 50 años. 
Radionoticieros, información politica y proceso electoral ...

\section{Cuadro 3}

Usuarios de radionoticieros por edad

(porcentajes)

\begin{tabular}{|l|c|c|c|c|}
\hline & $18-25$ & $26-35$ & $36-50$ & Mayor 50 \\
\hline Diciembre 1993 & 55,0 & 66,3 & 60,0 & 53,0 \\
\hline Agosto 1994 & 51,1 & 70,0 & 62,4 & 47,5 \\
\hline
\end{tabular}

Fuente: Sondeos diciembre 1993 y agosto 1994.

Con respecto al nivel de escolaridad de los entrevistados, los distintos sondeos presentan patrones de exposición muy contrastantes. En diciembre se observa un patrón harto homogéneo entre los distintos niveles, que registra una variación de solo tres puntos porcentuales entre el mayor y el menor. En contraste, en el sondeo de agosto se aprecian importantes diferencias. Por una parte, se hace evidente una relación entre nivel de escolaridad y grado de exposición. El índice mayor se encuentra entre quienes declararon escolaridad universitaria y posgrado, mismos que manifiestan un incremento de 10 puntos con respecto al sondeo anterior. En el otro extremo, quienes declararon una escolaridad de nivel básico experimentó una reducción de doce puntos. En este punto, se puede apreciar que la contienda electoral pudo haber influído en una mayor exposición y búsqueda de información radiofónica por parte de quienes declararon una mayor escolaridad.

\section{Cuadro 4}

Usuarios de radionoticieros por escolaridad (porcentajes)

\begin{tabular}{|l|c|c|c|c|}
\hline & Superior & Bachillerato & Secundaria & Primaria \\
\hline Diciembre 1993 & 58,9 & 57,8 & 58,1 & 61,0 \\
\hline Agosto 1994 & 68,3 & 62,2 & 57,4 & 49,0 \\
\hline
\end{tabular}

Fuente: Sondeos diciembre 1993 y agosto 1994.

Finalmente, con respecto a la ocupación nuevamente se expresan diferencias entre ambos sondeos. Aquí también, se aprecian patrones que tienden a la homogeneidad en el mes de diciembre, asimismo las variaciones que se manifiestan en el sondeo de agosto 
constituyen el elemento más interesante. En este caso, en los días previos a los comicios electorales, se aprecia un incremento en la exposición por quienes se declararon profesionistas, comerciantes y estudiantes, incremento que es particularmente notable entre los primeros. Por el contrario, la disminución se hizo presente entre los empleados, las amas de casa y quienes declararon otros empleos o no lo especificaron.

\section{Cuadro 5 \\ Usuarios de radionoticieros por ocupación \\ (porcentajes)}

\begin{tabular}{|c|c|c|c|c|c|c|}
\hline & Profesionista & Comerciante & Empleado & $\begin{array}{c}\text { Ama de } \\
\text { casa }\end{array}$ & Estudiante & Otras \\
\hline Diciembre 1993 & 57,8 & 53,8 & 60,8 & 60,6 & 55,8 & 60,0 \\
\hline Agosto 1994 & 71,4 & 57,7 & 54,1 & 53,7 & 61,8 & 53,8 \\
\hline
\end{tabular}

Fuente: Diciembre 1993 y agosto 1994

Junto a los patrones de exposición y la magnitud de la audiencia, los hábitos de consumo constituyen un indicador de la penetración que los radionoticieros alcanzan entre la radioaudiencia. La penetración de un medio informativo se encuentra en relación directa a la asiduidad conque sus usuarios lo consumen. En este punto en particular, los radionoticieros manifiestaron tener una gran penetración entre sus usuarios, superando inclusive a la prensa escrita que cuenta con mayor audiencia.

En efecto, en el sondeo de diciembre una porción claramente mayoritaria (76.5\%) de quienes declararon exponerse a los radionoticieros, aceptó escucharlos diariamente. Por lo tanto, si se considera que del total de la muestra (415) 186 entrevistados (44.8\%) se exponían diariamente a un informativo radiofónico, dicha proporción superaba ampliamente al $27.2 \%$ de quienes declararon exponerse diariamenta a la prensa escrita.

Esta alta penetración se confirma cuando el $37.7 \%$ de los radioescuchas afirmaron haberse expuesto al radionoticiero ese mismo día, y el $44.0 \%$ haberlo hecho el día anterior. Este dato, contemplado en el total de la muestra nos indica que el $52.7 \%$ de los entrevistados habían escuchado algun programa informativo el día de la aplicación de la encuesta ó el anterior. ${ }^{10}$

10 Hay que tomar en cuenta que la encuesta se aplicó un sábado, día que algunos radionoticieros no tienen emisión. 


\section{Gráfica 1}

- Frecuencia de exposición

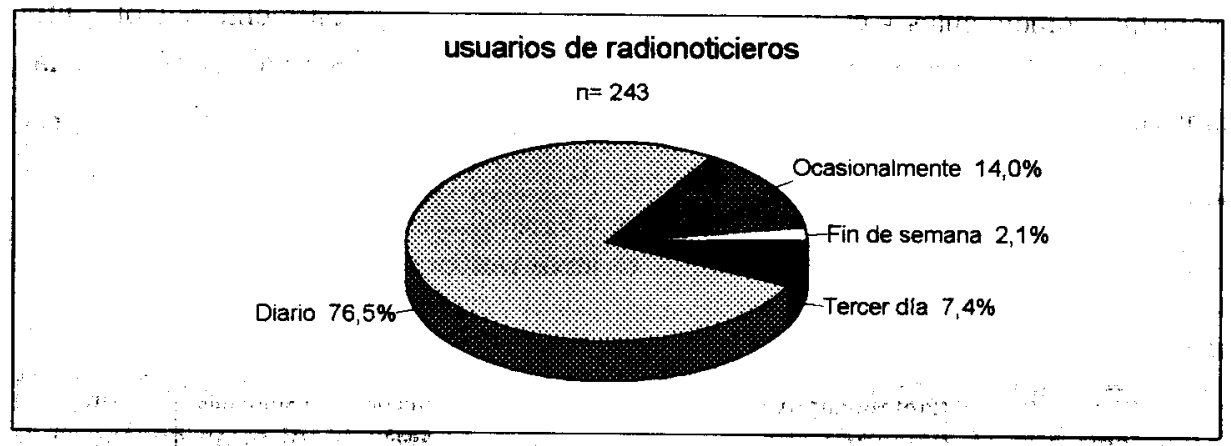

Con respecto a la hora en que se exponen a estos programas, la mayoría de los radio-oyentes señalaron como favorito al segmento comprendido entre las 7 y las 10 de la mañana, que corresponde al horario en que se transmitía la emisión principal de algunos radionoticieros (Gráfica 2). En los segmentos subsecuentes la exposición hacia éstos disminuye drásticamente llegando a su mínima expresión en el nocturno. Finalmente, un importante porcentaje de los radioescuchas (16.5\%) declararon exponerse en forma intermitente a los radionoticieros y no ajustarse a un horario establecido.

\section{Los radionoticieros preferidos por los tapatíos}

Del total de la oferta informativa que circulaba por las ondas radiofónicas, solamente seis opciones manifestaron tener alguna presencia en las preferencias de los radioescuchas. De entre ellas, los usuarios de este tipo de programa privilegiaron al radionoticiero Notisistema, seguido por la estación informativa Radio Metrópoli. Estas dos opciones -que pertenecen a la misma empresa- representaron el $47.7 \%$ y el $54.3 \%$ de las preferencias de los radioescuchas en los sondeos de diciembre y agosto, respectivamente. En menor medida, otras cuatro opciones informativas alcanzaron un cierto nivel de penetración. Las demás no lograron una presencia significativa.

Sin embargo, a lo largo del período analizado se observan importantes modificaciones en la penetración alcanzada por las diversas opciones informativas, a excepción de Notisistema que se mantiene invariablemente a la cabeza. En este lapso, los radionoticie- 
ros Para empezar y Norisat y la estación Radio Metrópoli muestran un importante crecimiento, en contraste con el radionoticiero Aquí Guadalajara-más noticias (XEDK) y la estación XEw que acusan una severa disminución.

\section{Gráfica 2}

Horario de exposición

Usuarios de radionoticieros

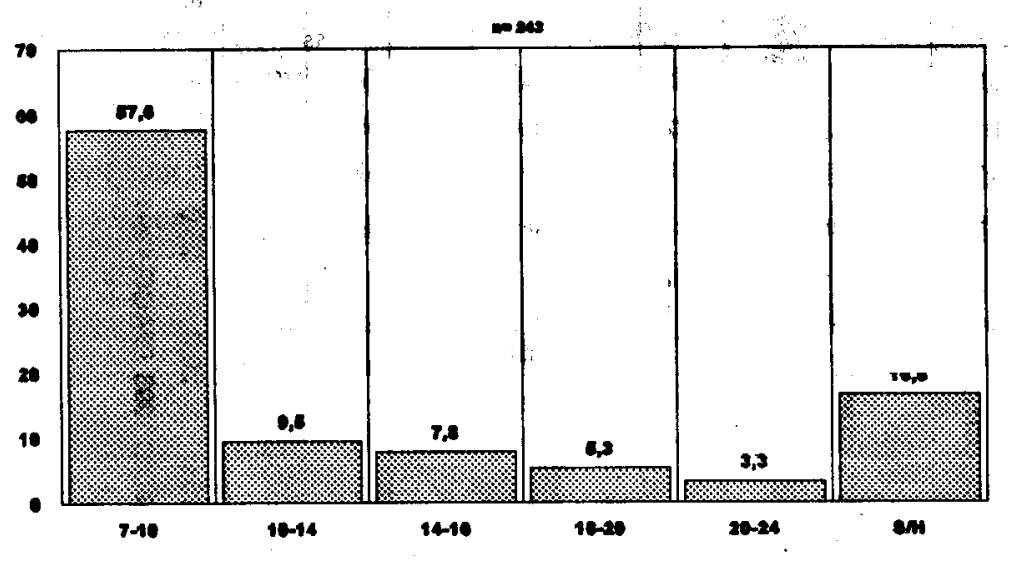

Fuente: Sondeo diciembre 1993.

\section{Radionoticieros y estrato social}

En el lapso estudiado, la relación entre la preferencia por cierto radionoticiero y la pertenencia a determinado estrato social presenta rasgos contradictorios, que van desde una cierta consistencia hasta bruscas modificaciones en sus preferencias hacia determinada opción informativa.

El primer caso lo ilustran Notisistema y Radio Metrópoli que mantienen conjuntamente la hegemonía en ambos sondeos. Destaca particularmente el crecimiento que experimenta Notisistema en el sondeo de agosto entre los radioescuchas de los estratos "A" y "B" llegando a constituir la opción mayoritaria, así como el incremento de Radio Metrópoli en los estratos "C" $\mathrm{y}$ "D". Un rasgo especial es que ambas opciones son -junto con Para Empezar- los únicos radionoticieros identificados con precisión por los 


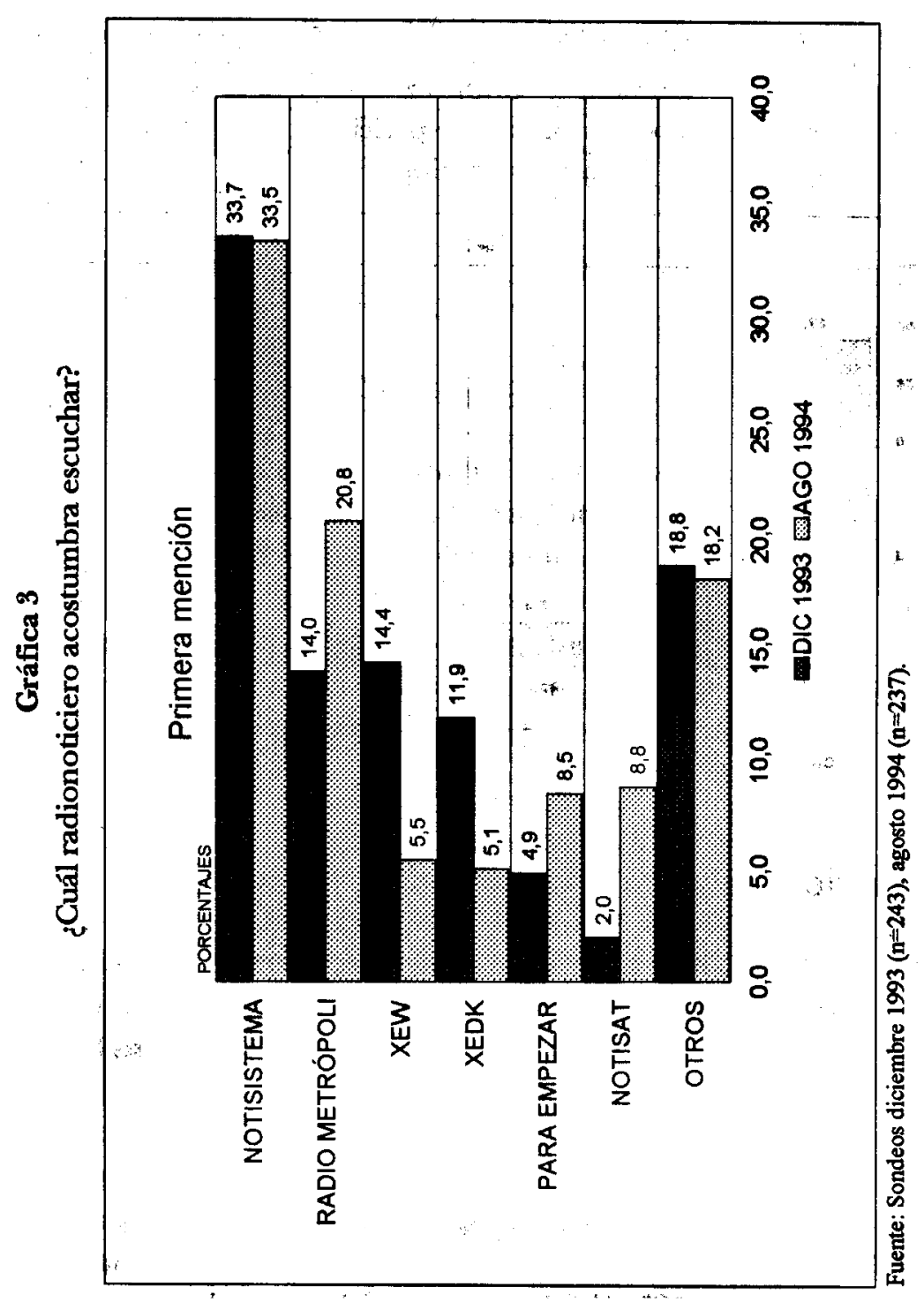


radioescuchas pertenecientes al estrato " $\mathrm{A}$ ", alcanzando en el mes de agosto a concentrar el $76.4 \%$ de las preferencias de dicho estrato.

En el segundo caso, se observa la abrupta disminución de las preferencias hacia XEW, que pierde su presencia en el estrato " $\mathrm{B}$ ", pero la mantiene casi en el mismo rango en el estrato " $\mathrm{C}$ " y también, aunque en menor proporción en el " $\mathrm{D}$ ", aunque en éste último es donde obtiene su mejor acogida. Asimismo, el detrimento de Más Noticias, que alcanzaba cierta presencia en el mes de diciembre entre los estratos " $C$ " y "D", en el sondeo de agosto solamente logra captar las preferencias de un escaso sector del estrato "D". En contraste, Para empezar que había sido mencionado por radioescuchas de los estratos "A", "B" y "C" en el primer sondeo, incrementó su presencia en el mes de agosto, y en el estrato "B" lo hizo triplicando su participación. En el caso de Notisat, que en diciembre había obtenido una presencia marginal en los estratos " $\mathrm{B}$ " $\mathrm{y}$ " $\mathrm{C}$ " misma que mantiene en agosto, acusó un crecimiento impresionante entre los radioescuchas del estrato " $\mathrm{D}$ ".

\section{Cuadro 6}

Los radionoticieros y su audiencia

Distribución por estrato social

\begin{tabular}{||l|c|c|c|c|c|c|c|c|}
\hline \multirow{2}{*}{} & \multicolumn{2}{|c|}{ A } & \multicolumn{2}{c|}{ B } & \multicolumn{3}{c|}{ C } & \multicolumn{2}{c|}{ D } \\
\cline { 2 - 10 } & 1993 & 1994 & 1993 & 1994 & 1993 & 1994 & 1993 & 1994 \\
\hline Notisistema & 41,7 & 58,8 & 34,8 & 53,6 & 39,4 & 24,7 & 30,3 & 30,3 \\
\hline Radio & 8,3 & 17,6 & 17,4 & 3,6 & 12,1 & 24,7 & 15,5 & 22,7 \\
Metrópoli & & & & & & & & \\
\hline Notisat & & & 4,6 & 3,6 & 4,5 & 6,8 & 0,7 & 12,6 \\
\hline XEDK & & & 4,3 & 3,6 & 10,6 & 2,7 & 14,1 & 7,6 \\
\hline Para empezar & 16,7 & 27,6 & $\mathbf{8 , 7}$ & 25,0 & 9,1 & 11,0 & 1,4 & 1,7 \\
\hline XEw & & & 13,0 & & 3,0 & 2,7 & 21,1 & 9,2 \\
\hline Otros & 33,3 & 5,9 & 17,4 & 10,7 & 21,2 & 27,4 & 16,9 & 16,0 \\
\hline
\end{tabular}

Fuente: Sondeos diciembre 1993 y agosto 1994.

\section{Radionoticieros y género}

En la relación entre preferencias por determinado producto informativo y género el único rasgo que dentro de sus variaciones presenta una mayor homogeneidad es la preponde- 
rancia de Notisistema. Con mayor presencia entre las preferencias del auditorio femenino en el mes de diciembre, situación que se invierte en agosto, en donde además obtiene un significativo crecimiento entre los radioescuchas varones. Un efecto contrario es el que experimenta Radio Metrópoli que acusa en ese mismo lapso, el incremento en las preferencias del sector femenino y el decremento en el masculino.

En el caso de las demás opciones se aprecian variaciones importantes, resultado del aumento o disminución de las preferencias obtenidas entre los radioescuchas. Por un lado, el crecimiento evidenciado por Notisat se registra en ambos géneros, ubicándose en el tercer sitio de las preferencias. Con las mismas caracteristicas se expresa el incremento de Para empezar, el cual se posiciona en el cuarto sitio. Por el otro, las caída en las preferencias sufrida por Más noticias y XEW se presenta con características similares, la diferencia estriba en que XEW se ubica el el quinto sitio entre las preferencias femeninas, mientras que el sector masculino la relega al último lugar.

\section{Cuadro 7}

Los radionoticieros y su audiencia

Distribución por género

\begin{tabular}{|c|c|c|c|c|}
\hline \multirow[t]{2}{*}{$\cdots \cdots$} & \multicolumn{2}{|c|}{ Femenino } & \multicolumn{2}{|c|}{ Masculino } \\
\hline & 1993 & 1994 & 1993 & 1994 \\
\hline Notisistema & 35,3 & 28,3 & 31,8 & 39,1 \\
\hline $\begin{array}{l}\text { Radio } \\
\text { Metrópoli } \quad \therefore\end{array}$ & 10,3 & 28,8 & $\begin{array}{ll}18,7 & \\
& 8.6\end{array}$ & 13,6 \\
\hline Notisat & 2,9 & 7,9 & 0,9 & 10 \\
\hline XEDK & 12,5 & 4,7 & 11,2 & 5,5 \\
\hline Para empezar & 2,9 & 7,9 & 7,5 & 9,1 \\
\hline XEW & 16,9 & 7,1 & 11,2 & 3,6 \\
\hline Otros & 19,1 & 17,3 & 18,7 & 19,1 \\
\hline
\end{tabular}

Fuente: Sondeos diciembre 1993 y agosto 1994.

\section{Radionoticieros y edad}

La profunda variabilidad que manifiesta la preferencia hacia determinados radionoticieros en relación con los grupos de edad, alimenta poderosamente la certeza de la dificultad de establecer patrones de exposición y hábitos de consumo homogéneos entre los usuarios 
de los radionoticieros. Los resultados obtenidos mediante la aplicación de sondeos a muestras con muchas similitudes -número, distribución de variables y lugar de residencia- ${ }^{11}$ pero a distintos sujetos y diferente fecha, parecen confirmar la imposibilidad de definir una constante entre el programa informativo y su radioaudiencia.

En efecto, de un sondeo a otro, las preferencias manifestadas por los radioescuchas de los diversos grupos de edad sufren modificaciones sustanciales. La continuidad en la preferencia manifestada por la porción de uno de los grupos, como en el caso de Notisistema en los radioescuchas de 36 a 50 años, constituye una notable excepción. En todo caso, lo que más se acerca a una constante es la proporción de entrevistados que no acertaron a identificar el radionoticiero preferido. También es posible advertir que a la abrupta caída de XEW correspondió, al menos en los segmentos mayores de 36 años, la emergencia de Notisat.

\section{Cuadro 8}

Los radionoticieros y su audiencia

Distribución por grupos de edad

\begin{tabular}{|l|c|c|c|c|c|c|c|c|}
\hline & \multicolumn{2}{|c|}{$18-25$} & \multicolumn{2}{c|}{$26-35$} & \multicolumn{2}{c|}{$36-50$} & \multicolumn{2}{c|}{ Más de 50 } \\
\hline Notisistema & 57,6 & 31,4 & 26,2 & 39,3 & 29,2 & 24,7 & 15,9 & 34,2 \\
\hline $\begin{array}{l}\text { Radio } \\
\text { Metrópoli }\end{array}$ & 6,1 & 15,7 & 13,1 & 16,1 & 18,1 & 30,1 & 20,5 & 18,4 \\
\hline Notisat & & 5,7 & 4,9 & & & & & \\
\hline XEDK & 6,1 & 7,1 & 14,8 & 7,1 & 1,4 & 12,3 & 2,3 & 10,5 \\
\hline Para empezar & 3,0 & 10,0 & 8,2 & 3,6 & 6,9 & 9,6 & & 10,5 \\
\hline XEW & 3,0 & 4,3 & 19,7 & 8,9 & 11,1 & 4,1 & 29,5 & 5,3 \\
\hline Otros & 24,2 & 25,7 & 13,1 & 17,9 & 15,3 & 16,4 & 25,0 & 18,4 \\
\hline
\end{tabular}

Fuente: Sondeos diciembre 1993 y agosto 1994

\section{Radionoticiero y escolaridad}

En contraste con la anterior variable, en el caso de la escolaridad es posible percibir algunos patrones que manifiestan cierta continuidad. Esto es particularmente evidente en los grupos de mayor nivel escolar. En ellos la supremacía de Notisistema en la preferen-

11 Ver anexo "Radiografia de la muestra". 
cias de sus radioescuchas es indiscutible, y junto con Radio Metrópoli alcanza en ambos sondeos la porción mayoritaria. El otro elemento que es posible advertir en ambos grupos es la brutal reducción que experimenta Más noticias en el lapso analizado, cuyo lugar es ocupado por Notisat en el caso de los radioescuchas con estudios de nivel superior y por Para empezar entre los de nivel de bachillerato. Por su parte, los grupos conformados por radioescuchas con niveles de escolaridad correspondientes a estudios de enseñanza media (secundaria) y básica (primaria), también manifiestan ciertas características de continuidad. En ellos también Notisistema y Radio Metrópoli obtienen los porcentajes mas altos de preferencia, pero, al contrario de los niveles arriba analizados, en ellos se mantiene la preferencia hacia Más noticias, al mismo tiempo que se incrementa su inclinación por Notisat. La modificación mas visible, es la disminución que sufre la XEW entre los de nivel básico, al perder puntos porcentuales en el lapso comprendido entre los dos sondeos.

Un dato adicional que resulta muy interesante, es que en ambos sondeos quienes declararon menor escolaridad resultaron ser quienes mejor identificaron el radionoticiero preferido. Esto es particularmente visible en el sondeo de agosto, cuando solamente un puñado de entrevistados no acertó en la identificación. Es posible inferir, que entre los hábitos de consumo de este segmento, existe una mayor interacción entre el radioescucha y el programa preferido que el que se observa en los demás.

\section{Cuadro 9}

Los radionoticieros y su audiencia

Distribución por escolaridad

\begin{tabular}{||l|c|c|c|c|c|c|c|c|}
\hline \multirow{2}{*}{} & \multicolumn{2}{|c|}{ Superior } & \multicolumn{2}{c|}{ Bachillerato } & \multicolumn{2}{c|}{ Secundaria } & \multicolumn{2}{c|}{ Primaria } \\
\cline { 2 - 9 } & 1993 & 1994 & 1993 & 1994 & 1993 & 1994 & 1993 & 1994 \\
\hline Notisistema & 37,2 & 42,9 & 46,2 & 37,3 & 41,0 & 24,3 & 21,3 & 33,3 \\
\hline Radio & 14,0 & 12,5 & 9,6 & 11,8 & 11,5 & 27,1 & 20,0 & 25,5 \\
Metrópoli & & & & & & & & \\
\hline Notisat & & 8,9 & 3,8 & 5,9 & 3,3 & 10,0 & & 9,8 \\
\hline XEDK & 9,3 & 1,8 & 11,5 & 2,0 & 9,8 & $7,1,1$ & 13,3 & 9,8 \\
\hline Para empezar & 9,3 & 12,5 & 7,7 & 15,7 & 3,3 & 2,9 & & 5,9 \\
\hline XEW & 7,0 & 3,6 & 1,9 & 3,9 & 8,2 & 4,3 & 30,7 & 9,8 \\
\hline Otros & 23,3 & 17,9 & 19,2 & 23,5 & 23,0 & 24,3 & 14,7 & 5,9 \\
\hline
\end{tabular}

Fuente: Sondeos diciembre 1993 y agosto 1994 


\section{Radionoticieros y ocupación}

Con respecto al tipo de ocupación reportado, las modificaciones que se observan en la distribución de sus preferencias, es diferente en cada uno de los segmentos. Entre los profesionistas se advierte una drástica variación concretizada en el incremento experimentado por Notisistema y Radio Metrópoli en el sondeo de agosto, así como de Notisat, crecimiento que contrasta con la brusca disminución de Para empezar que pierde entre ambos sondeos casi 17 puntos porcentuales. Entre los comerciantes también alcanzan una presencia mayoritaria Notisistema y Radio Metrópoli, en este caso contrastando con la total desaparición del radionoticiero de la XEDK que en el sondeo de diciembre representaba un segmento importante de radioescuchas, así como la sustancial pérdida de audiencia que registra la XEW.

El patrón mas consistente en relación de las preferencias hacia determinado radionoticiero y el tipo de actividad desarrollada se expresa entre los empleados, entre éstos se observa que con variaciones no significativas Notisistema y Radio Metrópoli concentran la mayoría de las preferencias, seguidos por XEDK. Las modificaciones se advierten en el crecimiento experimentado por Notisat y Para empezar, en contraste con el sustancial adelgazamiento de XEw. Por su parte, entre las amas de casa Notisistema acusa una disminución casi exactamente proporcional al aumento que recibe Radio Metrópoli. Asimismo la XEW exhibe una merma importante en las preferencias hacia ella. Por el contrario, Para empezar acusa un incremento notable. Entre el segmento conformado por los estudiantes lo significativo es la sensible reducción sufrida por Notisistema en el sondeo de agosto, junto el crecimiento que manifiesta Para empezar y la irrupción de XEW en las preferencias de los radioescuchas. Finalmente, el grupo integrado por quienes se dedican a otra actividad o no la especificó que representa un porcentaje menor al $6 \%$, manifiesta un comportamiento harto irregular en el que destaca el aumento entre las preferencias de Radio metrópoli y Notisat arrojado por el sondeo de agosto con respecto al anterior.

\section{La radiodifusión como medio de información política}

Específicamente, como medio utilizado para obtener información política, la radio se encuentra empatada virtualmente con la prensa escrita al obtener el $36.6 \%$ de las menciones en el sondeo de diciembre. Sin embargo, la aventaja por cinco puntos en el 
Cuadro 10

Los radionoticieros y su audiencia

Distribución por ocupación

\begin{tabular}{|c|c|c|c|c|c|c|c|c|c|c|c|c|}
\hline & \multicolumn{2}{|c|}{ Profesionista } & \multicolumn{2}{|c|}{ Comerciante } & \multicolumn{2}{|c|}{ Empleado } & \multicolumn{2}{|c|}{ Ama de casa } & \multicolumn{2}{|c|}{ Estudiante } & \multicolumn{2}{|c|}{ Otras } \\
\hline & 1993 & 1994 & 1993 & 1994 & 1993 & 1994 & 1993 & 1994 & 1993 & 1994 & 1993 & 1994 \\
\hline Notisistema & 26,9 & 40,0 & 28,6 & 50,0 & 31,5 & 35,6 & 33,8 & 20,0 & 58,3 & 41,2 & 26,7 & 14,3 \\
\hline $\begin{array}{l}\text { Radio } \\
\text { Metrópoli }\end{array}$ & 3,8 & 17,1 & 14,3 & 13,3 & 19,2 & 22,0 & 14,3 & 27,7 & 8,3 & 8,8 & 13,3 & 35,7 \\
\hline Notisat & 3,8 & 11,4 & 3,6 & 6,7 & $1 ; 4$ & 8,5 & 2,6 & 7,7 & $y^{2}$ & 2,9 & & 28,6 \\
\hline XEDK & 7,7 & 2,9 & 14,3 & & 11,0 & 8,5 & 10,4 & 46 & 8,3 & 5,9 & & 7,1 \\
\hline $\begin{array}{l}\text { Para } \\
\text { empezar }\end{array}$ & 19,2 & $\begin{array}{l}2,9 \\
2\end{array}$ & 3,6 & 10,0 & 2,7 & 8,5 & 1,3 & 9,2 & $\overline{8,3}$ & 14,7 & 6,7 & 14,3 \\
\hline XEW & 11,5 & 5,7 & 17,9 & 3,3 & 15,1 & 5,1 & 19,5 & 7,7 & & 5,9 & 6,7 & \\
\hline Otros & 26,9 & 20,0 & 17,9 & 16,7 & 19,2 & 11,9 & 18,2 & 23,1 & 8,3 & 20,6 & 46,7 & 14,3 \\
\hline
\end{tabular}

Fuente: Sondeos diciembre 1993 y agosto 1994.

rubro de "mejor información", pero a su vez, es superada en lo que se refiere a la amplitud de la información (18.3\%) y a la veracidad (16.1\%) que le conceden los entrevistados. ${ }^{12}$

El nivel de importancia otorgado a la información difundida por la radio, marca con meridiana claridad sus estrechos límites, al observar que solamente el $15.4 \%$ de los entrevistados (421) en el mes de abril, la señaló como el medio de comunicación que ofreció la información más completa sobre las campañas presidenciales, proporción que desciende hasta el $10.9 \%$ respecto al asesinato de Luis Donaldo Colosio. En ambos casos la radio fué superada, aunque por estrecho margen, por la prensa escrita y en forma inobjetable por la televisión, que fué seleccionada por el $78.5 \%$ y el $81.9 \%$ de los entrevistados, respectivamente. ${ }^{13}$

Pero tal vez el índice mas claro de su papel marginal en la configuración del entorno político, se aprecia en que al ser consultados acerca de los medios a través de los cuales habían recibido la información que les permitió atribuir a los principales contendientes características tales como: democrático, populista, autoritario, etc..., la radio obtuvo solamente un $8.6 \%$ de menciones, siendo superada inclusive por la familia que alcanzó

12 Sondeo diciembre 1993.

13 Sondeo abril 1994. 
el $15.4 \%$ y quedando muy por debajo de los otros medios masivos como la prensa escrita $(21.9 \%)$ y la televisión $(32.5 \%){ }^{14}$

\section{Valoración de la información política}

Este papel marginal otorgado a la radiodifusión se corresponde con la exigua valoración que sus usuarios le conceden como suministrador de información política. Así, al ser interrogados acerca del medio que utilizan para obtener este tipo de información, solo un poco más de la mitad de los usuarios de Para empezar, Radio Metrópoli y Notisistema mencionaron a la radiodifusión. En los demás casos el porcentaje resultó inferior, llegando al extremo de Aquí Guadalajara-más noticias, al que solamente señaló la cuarta parte de sus adictos.

De igual manera, consultados acerca de la calidad de la información politica recibida, solamente la mitad de los usuarios de Radio Metrópoli y de Para empezar señalaron a la radio como el medio que ofrecía la "mejor" información, en tanto solamente la tercera parte de quienes se exponían a Notisistema y Más noticias repetiría en ningun otro rubrofue el que la totalidad de los partidarios de Notisat, lo evaluaron como el que suministró la "mejor" información. Sin embargo, respecto a la evaluación de la información radiofónica como las "más completa", solamente un poco más de la mitad de los usuarios de Notisat aceptó otorgar dicha valoración. Igualmente, con relación a la credibilidad que se otorga a las informaciones transmitidas por los medios, son los usuarios de Notisat quienes concedieron la mayor calificación a la radio. En los demás casos solamente la cuarta parte de los usuarios lo hace. El extremo en este caso, lo representan los partidarios de Más noticias, de los cuales ninguno consideró a la radio como el medio mas veraz.

En contraste, con respecto al tópico de la libertad de expresión los usuarios de los diversos radionoticieros expresan una opinión positiva en lo referente a la existencia de dicha libertad, aunque se aprecian significativas diferencias entre ellos, que se expresan en el $76.9 \%$ de los usuarios de XEW frente al $50.0 \%$ concedido por los adictos a Para empezar. Esta actitud favorable se convierte en escepticismo al ser consultados acerca de su puesta en práctica por parte de los medios, cuestión a la que solamente la cuarta parte de los usuarios de Para empezar respondió en forma afirmativa, en el otro extremo,

14 Sondeo agosto 1994. 
Radionoticieros, información política y proceso electoral ...

solamente un poco mas de la mitad los partidarios de Notisistema declararon que dicha libertad se ejercía prácticamente.

\section{Cuadro 11}

Valoración de la información política

Radioescuchas

\begin{tabular}{|l|c|c|c|c|c|c|}
\hline & Notisis. & Metróp. & Notisat & XEDK & Para emp. & XEW \\
\hline Como fuente* & 52,4 & 52,9 & 40,0 & 27,3 & 58,3 & 45,7 \\
\hline Mejor información* & 30,5 & 52,9 & 100 & 36,4 & 50,0 & 25,7 \\
\hline Más completa* & 19,5 & 35,3 & 60,0 & 9,1 & 33,3 & 25,7 \\
\hline Más veraz* $^{*}$ & 24,4 & 26,5 & 40,0 & 0,0 & 25,0 & 28,6 \\
\hline Libertad existe** & 64,6 & 67,3 & 57,1 & 66,7 & 50,0 & 76,9 \\
\hline Libertad se ejerce** & 55,7 & 44,9 & 42,9 & 41,7 & 25,0 & 46,2 \\
\hline
\end{tabular}

Fuente: Sondeos diciembre 1993* y agosto 1994**

\section{Valoración de la información sobre las campañas electorales}

Al ser consultados acerca del tratamiento que otorgaron los medios a los principales contendientes, se advierte una amplia y significativa coincidencia entre los radioescuchas de los diversos noticieros. En efecto, en forma mayoritaria los entrevistados señalaron que el candidato priista recibió el mejor tratamiento. También coincidieron, aunque en menor proporción, en señalar a Cárdenas como el contendiente peor tratado. La situación de Diego Fernández, que recibió menciones mínimas, presenta un cierto equilibrio tendiendo más hacia una voloración positiva, a excepción de los usuarios de Para empezar que lo colocan a solo diez puntos de Cárdenas como el peor tratado. Un caso especial que merece un análisis particular es la opinión emitida por los usuarios de XEW. Entre ellos la elección de Zedillo como el mejor tratado por parte de los medios, alcanzó el máximo porcentaje, pero además, no otorgaron ninguna mención, en este rubro, a los otros candidatos. En contraste, ninguno señaló a candidato priísta como el peor tratado, calificativo que recibieron en la misma proporción los otros candidatos. Un dato adicional es que entre éstos radioescuchas es donde Cárdenas recibe el resultado menos negativo. 
Lo interesante radica en el hecho de que XEW forma parte de la división radiofónica del consorcio Televisa, mismo que adoptó una política informativa muy peculiar, en la cobertura que realizó de las campañas políticas. Basada en un modelo que se apegaba a un cierto patrón de objetividad -cobertura similar a todas las campañas-, no obstante fue utilizado para apuntalar sus preferencias partidistas. El resultado analizado constituye una muestra del carácter exitoso del procedimiento.

\section{Exposición a los radionoticieros e interés por asuntos políticos}

Utilizando la exposición a los medios como indicador, es posible obtener una aproximación al interés que manifiestan sus usuarios hacia los asuntos políticos. Un índice que permite detectar dicho interés consiste en la identificación positiva por parte de sus usuarios de determinados personajes ó acontecimientos vinculados al ámbito de la política. Para este propósito se analizará la identificación positiva de la fecha de los comicios y de los principales contendientes a la presidencia.

En el primer caso, se advierte que a pesar de haberse determinado con varios meses de antelación al 21 de agosto como la fecha en que se realizaria la jornada electoral, en el sondeo de diciembre solamente un escaso porcentaje de los usuarios de los radionoticieros la identificaron positivamente. Para el mes de abril, aunque la proporción había aumentado significativamente, todavía existía un considerable margen de radioescuchas que desconocía la fecha de los comicios electorales. Un dato interesante, que muestra cierta consistencia es que esta brecha se ensanchaba entre quienes manifestaban preferencia hacia las "estaciones informativas", y se hacía más reducida entre quienes se declaraban exponerse a algún radionoticiero. Asi se aprecia que los que manifestaron mayor desconocimiento fueron los usuarios de XEW y Radio Metrópoli, en contraste, quienes se adscribireron como oyentes de algún radionoticiero, manifestaron mayor nivel de información, llegando al caso de que la totalidad de entrevistados que se inclinaron por Notisat, realizaron la identificación correctamente. De donde podría inferirse una correlación positiva entre exposición a radionoticieros e interés sobre asuntos políticos. La excepción la representan los usuarios de Aqui Guadalajara que siendo radionoticero obtuvo el índice mas bajo en identificación positiva. (Gráfica 10) 
Radionoticieros, información politica y proceso electoral ...

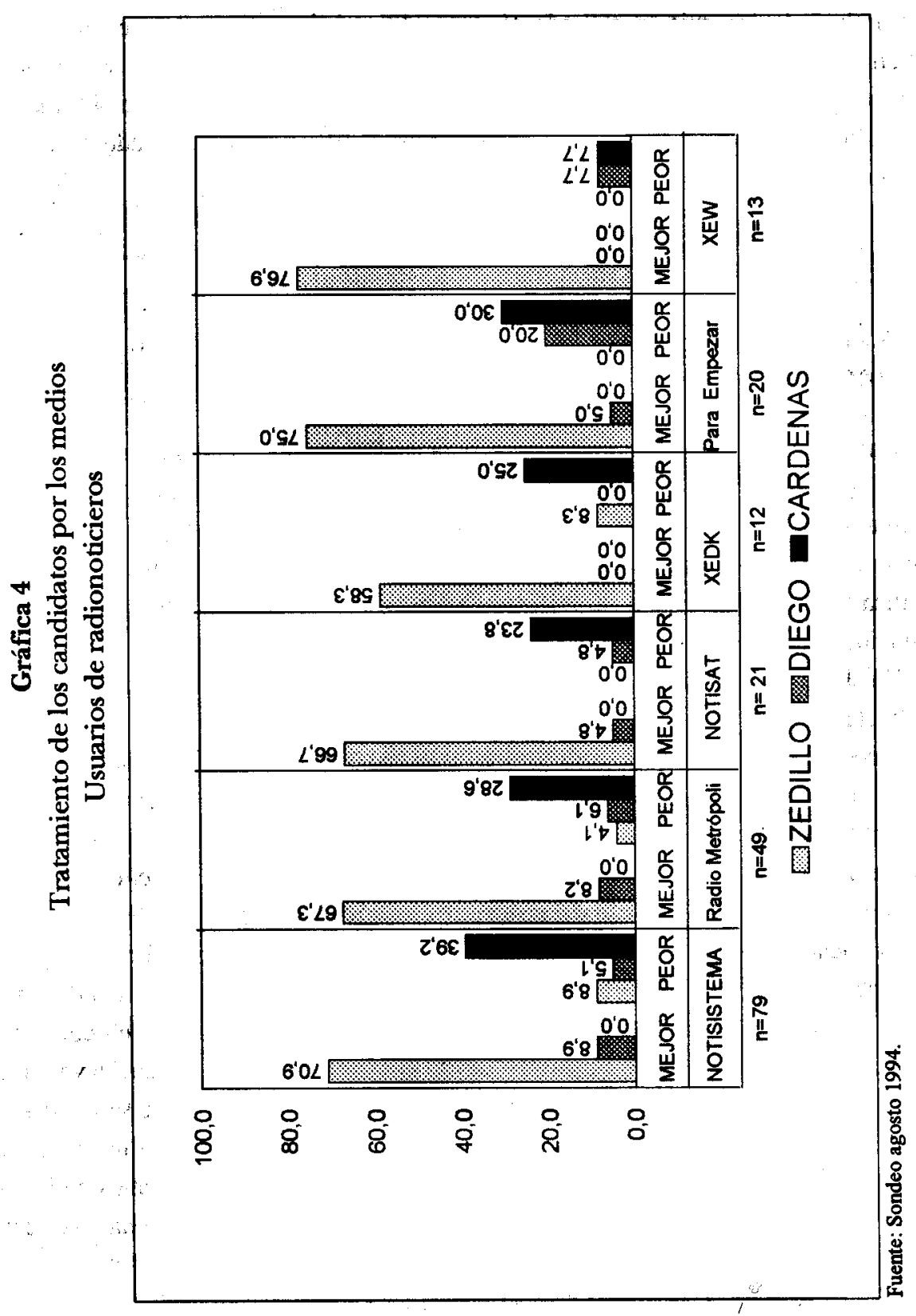


Otro indicador muy pertinente para medir el nivel de interés que el fenómeno electoral despertó entre la radioaudiencia consiste en la identificación positiva de los candidatos presidenciales que resultaron nominados por los tres partidos de mayor influencia entre el electorado. En este aspecto, dos son los fenómenos que presentan cierta consistencia a lo largo del proceso. El primero, es que en todos los sondeos, el candidato priísta fue el más identificado.

En efecto, no obstante de haber sido el último en recibir su nominación -en ser destapado- como candidato a la presidencia, Luis Donaldo Colosio, candidato del PRI resultó ser el conocido por los radioescuchas en el sondeo de diciembre. Igualmente, en el sondeo de abril, a escasos dias de haberse dado a conocer la candidatura de Zedillo el grueso de los radioadictos lo identificaron positivamente, llegando dicha identificación, en lo días previos a la jormada electoral, a superar el 90 por ciento. El contraste se advierte en los candidatos opositores, en donde se aprecia que Cárdenas, que había sido identificado positivamente en segundo lugar en el sondeo de diciembre, experimenta un crecimiento limitado, siendo rebasado por el candidato panista en el sondeo de agosto. Es precisamente en el caso de Diego Fernández de Cevallos donde se aprecia el cambio mas drástico al pasar de casi el 20 por ciento en diciembre a más del 80 en agosto. Obviamente, tal incremento respondía a las preferencias electorales de los tapastíos, cuestión que la jornada electoral se encargó de evidenciar, al superar ampliamente el candidato panista a los otros contendientes.

El segundo fenómeno que se aprecia es la ausencia de un patrón consistente entre la exposición a determinado radionoticiero y la identificación positiva de los candidatos. Aunque en algunos casos se observa un incremento sostenido a lo largo del proceso, respecto a los tres candidatos como en Notisistema, Radio Metrópoli, Para empezar y $\mathrm{XEW}$, se aprecian no obstante diferencias significativas entre ellos. En los extremos estarian, por un lado, Para empezar cuyos usuarios manifestaron el mayor nivel de información, llegando al grado de identificar positivamente a los tres contendientes en los sondeos de abril y agosto. Por el otro, los usuarios de XEW que obtuvieron el nivel mas bajo en el sondeo de diciembre, acusan en el proceso un crecimiento espectacular. Sin embargo, en otros casos -Notisat y XEDK-presentan variaciones importantes respecto a la identificación de los candidatos a lo largo del proceso. En este caso se aprecia nuevamente que los usuarios de radionoticieros superaron con respecto a la identificación de candidatos a quienes declaron exponerse a las estaciones informativas 


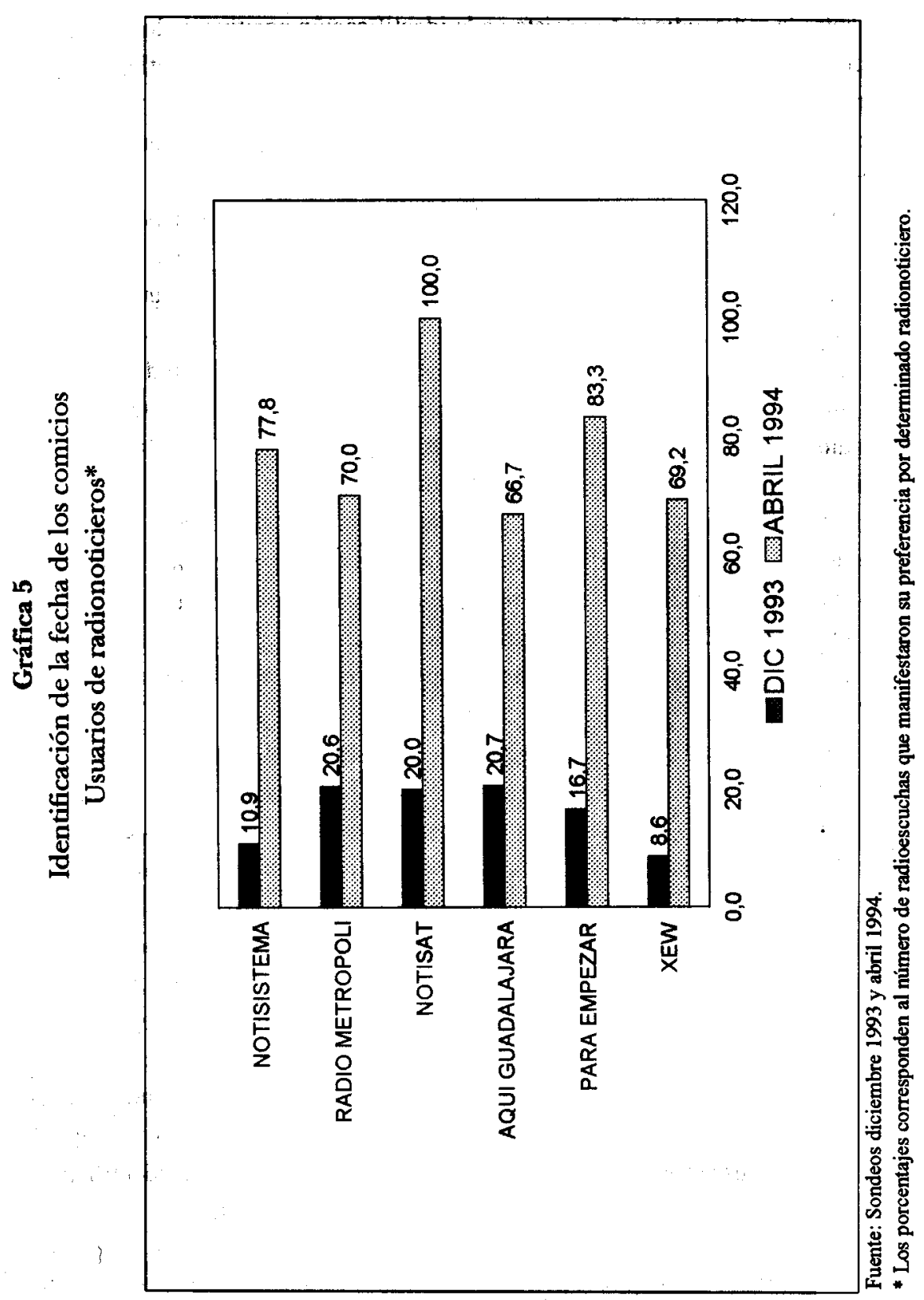




\section{Cuadro 12}

Identificación de los candidatos

Radioescuchas

\begin{tabular}{|c|c|c|c|c|c|c|}
\hline & Notisist. & Metropo. & Notisat & XEDK & Para emp. & XEW \\
\hline & \multicolumn{6}{|c|}{ PRI } \\
\hline Diciembre 1993 & 72,0 & 82,4 & 60,0 & 63,6 & 91,7 & 54,3 \\
\hline Abril 1994 & 85,7 & 94,0 & 50,0 & 100 & 100 & 84,6 \\
\hline \multirow[t]{2}{*}{ Agosto 1994} & 92,4 & 95,9 & 90,5 & 83,3 & 100 & 100 \\
\hline & \multicolumn{6}{|c|}{$\mathbf{P A N}$} \\
\hline Diciembre 1993 & 22,0 & 420,6 & 40,0 & 18,2 & 41,7 & 8,6 \\
\hline Abril 1994 & 61,9 & 60,0 & 25,0 & 60,0 & 100 & 46,2 \\
\hline \multirow[t]{2}{*}{ Agosto 1994} & 83,5 & 87,8 & 81,0 & $66,7:$ & 100 & 92,3 \\
\hline & \multicolumn{6}{|c|}{ PRD } \\
\hline Diciembre 1993 & 42,7 & 52,9 & 60,0 & 36,4 & 91,7 & 40,0 \\
\hline Abril 1994 & 63,6 & 76,0 & 25,0 & 60,0 & 100 & 69,2 \\
\hline Agosto 1994 & 81,0 & 79,6 & 76,2 & 66,7 & 100 & 84,6 \\
\hline
\end{tabular}

Fuente: Sondeos diciembre 1993, abril y agosto 1994.

Sin embargo, un dato que resulta altamente revelador del interés que los radioescuchas otorgaron al proceso electoral, indudablemente el evento político de mayor transcendencia en la vida nacional, es que solamente un porcentaje aproximado al $80 \%$ de los usuarios identificaron correctamente a los tres principales contendientes a la primera magistratura. Es decir, que dos de cada diez entrevistados que afirmaron exponerse a cualquiera de los radionoticieros o estaciones informativas, demostraron no disponer de una información, que en la víspera de los comicios saturaba, literalmente, no sólo los espacios informativos de los medios de comunicación, sino las calles, las paredes, los postes y las conversaciones.

Finalmente, consultados acerca de su ubicación en el espectro político (izquierda, centro, derecha) el grueso de los entrevistados se resistió a definirse por cualquiera de ellas. En casi todos los casos, la mitad o más se declararon sin orientación política. En el caso de Notisat y XEDK, la proporción de los indefinidos superó el $70 \%$. Entre lo que definieron su postura, un porcentaje que fluctuaba por el $25 \%$ se ubicó en una posición centrista, a excepción de los usuarios de Notisat (14.3\%) los de XEDK (8.3\%). Entre quienes se ubicaron a la derecha, manifestaron en proporción similar -alrededor del 15\%- 
su preferencia hacia Notisistema, Radio Metrópoli, Para empezar y XEW, seguida por XEDK (8.3\%) pero ninguno seleccionó a Notisat, que a su vez, recibió el mayor porcentaje de los partidarios de la izquierda (14.3\%), seguida por Para empezar (10.0\%). En contraste, su inclinación disminuyó drásticamente en el caso de Notisistema (5.1\%) y Radio Metrópoli (2.0\%). De esta manera, los usuarios de Notisistema y Radio Metrópoli se ubican en una posición decididamente inclinada hacia la centro-derecha; los usuarios de Para empezar y XEW, aunque ubicados mayoritariamente en la misma posición, también cuentan en su radioaudiencia con segmentos significativos de la izquierda; en el caso de Aqui Guadalajara, sus usuarios de distribuyen equitativamente entre las posiciones del espectro; finalmente, la totalidad de los usuarios de Notisat se identificaron como de centro-izquierda.

\section{Conclusiones}

De lo antes expuesto de desprenden algunas consideraciones que dibujan en sus rasgos más generales, lo patrones de exposición de la radioaudiencia tapatía y su comportamiento en el marco de un proceso electoral.

Un primer rasgo tiene que ver con la exigua magnitud de sus usuarios. El estudio constata que la audiencia hacia la información radiofónica, apenas rebasa la mitad $\mathbf{- 5 7 . 8 \%}$ promedio-del auditorio potencial.

Otro rasgo es que una proporción superior al ochenta por ciento de esta radioaudiencia, concentra su preferencia en solamente seis de la opciones informativas. De entre ellas, destacan como las mas favorecidas el radionoticiero Notisistema y la estación informativa Radio Metrópoli, ambos generados en la localidad y pertenecientes a la misma empresa: Operadora Unidifusión S.A. de C.V.

Otro rasgo consiste en la clara preferencia de los usuarios de la información radiofónica a exponerse mayoritariamente (57.6\%) en el horario comprendido entre las 7 y las 10 de la mañana, así como de la existencia de un significativo sector de la radioaudiencia que declararon no ajustarse a un horario definido, carácterística peculiar de los adictos a la radiodifusión.

Un hallazgo interesante resultó de la comparación de los patrones de exposición observados en los sondeos de diciembre y agosto. Estos sondeos, aplicados a muestras en condiciones harto similares -tamaño, distribución por variables, lugar de residenciapero a distintos sujetos y en fechas diferentes, arrojaron patrones que fluctúan entre la 
consistencia y la variabilidad. Así, en lo que respecta a la exposición a la información radiofónica, se aprecia que la variabilidad parece ser la característica dominante. En efecto, a excepción de la variable de género en la que se observa cierta consistencia, en todos los demas casos lo que rige en el comportamiento de los radioescuchas de uno y otro sondeo es la variación. Dicha modificación se observa tanto en el patrón manifestado por las variables en ambos sondeos, como entre los segmentos que integran estas mismas variables. En contraste, con respecto a las preferencias se observa una mayor consistencia. En ambos sondeos Notisistema y Radio Metrópoli encabezaron las opciones informativas y refendaron su hegemonía, en particular Notisistema.

Otro hallazgo de una relevancia central para este estudio consistió en descubrir que la cercanía del evento electoral no se reflejó en un aumento hacia la exposición de informativos radiofónicos. Sin embargo, si se detectaron modificaciones en los patrones de exposición que parecen haber sido motivadas por el cacontecimiento político. Así se observa un incremento en la búsqueda de información radiofónica entre los usuarios de mayores ingresos, de género masculino, que fluctuaban entre los 26 y 50 años de edad, que declararon un mayor nivel de escolaridad y que se ubicaron en las actividades de profesionista, comerciante y estudiante.

Sin embargo, a pesar de que la radiodifusión se encuentra entronizada entre los medios utilizados para la obtención de informaciones, la transcendencia que obtiene como vehículo de información política es bastante raquítica. En efecto, la importancia que otorgan sus usuarios en la conformación de sus conocimientos y opiniones políticas es sumamente marginal. Este carácter limitado se expresa en el hecho de que apenas un número ligeramente superior a la mitad de los usuarios de tres radionoticieros, declararon utilizarlos como para obtener información acerca de acontecimientos políticos. Y a excepción de Notisat, que resultó el mejor evaluado por sus usuarios, los demás informativos radiofónicos obtuvieron una evaluación bastante crítica respecto a la calidad de información suministrada y de su veracidad. Pero el indicador más claro de su escasa influencia surge de la exigua proporción de usuarios que la señalaron como el medio utilizado para caracterizar a los candidatos presidenciales.

Resulta evidente, entonces, que como medio de información política, la radiodifusión se encuentra a la zaga de la prensa escrita y la televisión.

Otro hallazgo valioso consistió en la valoración que los radioescuchas hicieron sobre el tratamiento noticioso de la contienda electoral. Aqúi, destaca la coincidencia general sobre el tratamiento preferencial y positivo que los medios de comunicación otorgaron 
Radionoticieros, información política y proceso electoral ...

al candidato del PRI. Coinciden también en señalar a Cuauhtémoc Cárdenas como el peor tratado.

Asimismo los datos obtenidos confirman que la exposición a los programas informativos no necesariamente está vinculada al interés que sus usuarios profesan por los asuntos públicos. En efecto, resulta harto ilustrativo el hecho de que hacia el mes de abril un considerable porcentaje de radioescuchas desconocían la fecha de los comicios electorales. Pero el hallazgo que sustenta esta afirmación de manera inobjetable, es el de que en vísperas del 21 de agosto, a excepción de los usuarios de Para empezar, un porcentaje significativo de radioescuchas no fué capaz de identificar correctamente a los candidatos de los tres principales partidos.

Finalmente, aunque en forma mayoritaria los entrevistados se mostraron reticentes a identificarse con alguna de las tendencias del espectro político, no obstante, es posible advertir cierta correspondencia entre la preferencia hacia determinado radionoticiero y la inclinación política del radioescucha. En este sentido, Notisistema y Radio Metrópoli resultaron favorecidos por quienes se ubicaron en una posición de centro-derecha; Notisat por su parte, resultó el preferido de quienes se alinearon con la centro-izquierda. 


\section{Anexo}

\section{Vitrina metodológica}

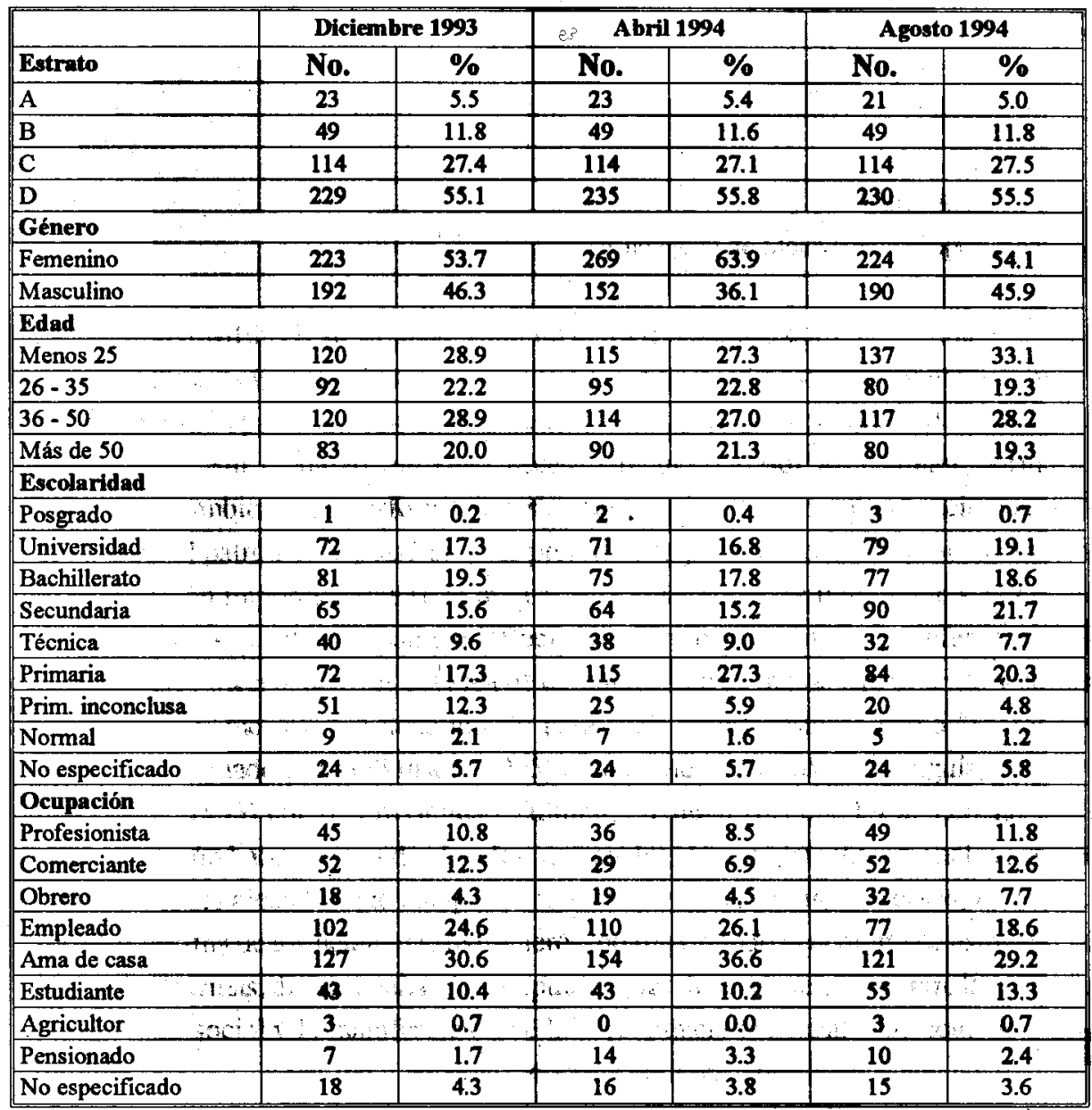

Sondeos: Diciembre $1993(\mathrm{~N}=415)$; abril $1994(\mathrm{~N}=421)$; agosto $1994(\mathrm{~N}=414)$

Tipo de muestreo: Probabilístico estratificado por cuotas

Universo de estudio: Población de 18 años y más de la zona metropolitana de Guadalajara

Lugar de levantamiento: Vivienda del entrevistado

Confiabilidad estadística: $95 \%$

Grado de error: $+/-5 \%$ 